\title{
Superstring amplitudes, unitarity, and Hankel determinants of multiple zeta values
}

\author{
Michael B. Green ${ }^{a, b}$ and Congkao Wen ${ }^{a}$ \\ ${ }^{a}$ Centre for Research in String Theory, School of Physics and Astronomy, \\ Queen Mary University of London, \\ Mile End Road, London E1 4NS, U.K. \\ ${ }^{b}$ Department of Applied Mathematics and Theoretical Physics, University of Cambridge, \\ Wilberforce Road, Cambridge CB3 OWA, U.K. \\ E-mail: M.B.Green@damtp.cam.ac.uk, c.wen@qmul.ac.uk
}

ABSTRACT: The interplay of unitarity and analyticity has long been known to impose strong constraints on scattering amplitudes in quantum field theory and string theory. This has been highlighted in recent times in a number of papers and lecture notes. Here we examine such conditions in the context of superstring tree-level scattering amplitudes, leading to positivity constraints on determinants of Hankel matrices involving polynomials of multiple zeta values. These generalise certain constraints on polynomials of single zeta values in the mathematics literature.

Keywords: Effective Field Theories, Scattering Amplitudes, Superstrings and Heterotic Strings

ArXiv EPrint: 1908.08426 


\section{Contents}

1 Introduction and outline 1

2 Unitarity constraints on low-energy expansion coefficients 3

2.1 The Stieltjes half-moment sequence 6

$\begin{array}{lll}2.2 & \text { Positivity conditions on moments of the amplitude } & 7\end{array}$

3 Four-particle open superstring tree amplitudes $\quad 9$

3.1 Low-energy expansion of the four-particle open superstring tree amplitude 10

$4 \quad$ Four-particle closed superstring tree amplitudes $\quad 13$

$\begin{array}{lll}4.1 & \text { The closed-string low-energy expansion coefficients } & 15\end{array}$

$\begin{array}{lll}4.2 & \text { Low-energy expansion of } \tilde{A}_{c l}^{(s)}(s, t) & 16\end{array}$

5 Discussion $\quad 18$

$\begin{array}{ll}\text { A Four-particle superstring tree amplitudes at higher orders in } t & 20\end{array}$

\section{Introduction and outline}

The S-matrix programme of the 1960s demonstrated that a number of very striking properties of scattering amplitudes arise from a few powerful principles, such as unitarity and analyticity. A key insight that arose from the analysis of hadronic experiments around 1967 is the idea of duality in hadronic processes - the sum of an infinite number of resonance poles in the $s$-channel reproduces the sum of an infinite set of poles in the $t$-channel. This is the "dual resonance" realisation of the bootstrap programme and was an essential feature of the Veneziano model [1], which evolved into the bosonic open-string theory, and the Virasoro model [2], which evolved into the bosonic closed-string theory. It is also a feature of large- $N$ limit of $\mathrm{SU}(N)$ QCD [3]

In recent years there has been a revival of these ideas in the context of important developments in quantum field theories related to the Standard Model and quantum gravity, as well as their supersymmetric counterparts. Unitarity together with analyticity lead to nontrivial conditions on low-energy effective field theory that imply that Wilson coefficients should respect certain positivity bounds [4]. Such positivity bounds have many important applications ranging from ruling out phenomenological models [4] to proving the $a$-theorem in four dimensions [5], and to a better understanding of the weak gravity conjecture [6] (see e.g. [7-10]). The results of [4] have recently been extended to an infinite set of positivity 
conditions on these coefficients by Arkani-Hamed, Huang and Huang, as described in [1113]. ${ }^{1}$ Recent applications of these ideas can be found in [16], which explores constraints on low-energy spectrum of quantum field theory when coupled to gravity, as well as implications for the weak gravity conjecture. These ideas have also been applied to correlation functions in conformal field theories [17] (see also [18]).

Some background material will be reviewed in section 2, following closely the ideas in [11-13]. A general consequence of unitary and analyticity in the Mandelstam invariants $(s, t, u)$ that follows from simple dispersion relations is that coefficients in the low energy expansion of the four-particle amplitude are constrained by ultraviolet properties of the amplitude. These coefficients can be assembled into Hankel matrices ${ }^{2}$ that must generally be totally non-negative - i.e. all minors of such matrices are non-negative. For theories that exhibit duality, such as string theory or large- $N$ QCD, which contain an infinite number of particle states with unbounded masses and spins, the coefficients of the Hankel matrices are necessarily totally positive. These constraints follow from the fact that the low energy coefficients form a Stieltjes half-moment sequence [19]. The precise statement of these constraints on the low-energy coefficients depends on properties of the ultraviolet behaviour of the amplitude. Furthermore, there are particular subtleties in the general analysis of theories with massless particles, which have massless threshold branch cuts in the complex $s$-plane and are also singular in the forward direction [15].

This paper explores these constraints on the low-energy expansion of tree amplitudes describing the scattering of four massless particles in open and in closed superstring theories that illustrate these points. Since we restrict our considerations to tree amplitudes we will avoid dealing with the subtlety of massless thresholds. Furthermore, we will see that there is no problem in subtracting the massless exchange contributions that are singular in the forward direction.

Clearly there is a limited amount of information that can be obtained by considering only massless four-particle tree amplitudes. For example, in order to sample all the information contained in the no-ghost theorem in string theory [20-22] it would be necessary to consider amplitudes with arbitrary numbers of massless external scattering particles, or to consider all four-particle amplitudes with arbitrary massless and massive external states.

Nevertheless the information in massless four-article tree amplitudes has some direct connections with mathematical considerations. As is well-known, in the case of the open superstring the coefficients in the low-energy expansion are rational polynomials in Riemann zeta values. As will be seen in section 3 , this leads to positivity conditions on determinants of Hankel matrices of zeta values that extend those discussed in [23, 24].

In section 4 we will see that the situation is more subtle in the closed-string case. In this case the Hankel matrix constraints do not apply to the low-energy expansion of the full four-particle amplitude at fixed $t$ due to negative contributions from the $u$-channel poles. In order to analyse the unitarity constraints we will separate the fixed- $t$ amplitude into the

\footnotetext{
${ }^{1}$ Different kinds of improved positivity bounds on low-energy coefficients of EFTs that generalise the results of [4] were explored in $[14,15]$.

${ }^{2} \mathrm{~A}$ Hankel matrix is a $m \times n$ matrix $H[a]_{i j}$ in which $a_{i j}$ depends only on $i+j$. For our purposes it is sufficient to consider square Hankel matrices for which $m=n$.
} 
sum of the contribution of $s$-channel poles and the contribution of $u$-channel poles. The low energy expansion of the contribution from the $s$-channel poles contains a combination of irreducible Multiple Zeta Values (MZVs) ${ }^{3}$ which cancel in the full four-particle amplitude (resulting in coefficients that are rational polynomials of odd zeta values). We will argue that the unitarity conditions lead to total positivity of the Hankel matrices built out of these MZVs. This gives a host of conditions on rational polynomials of particular combinations of MZVs.

These results are summarised and discussed in section 5 .

\section{Unitarity constraints on low-energy expansion coefficients}

In this section we will follow the discussion in [11-13] (see also [16]). We begin by reviewing the general arguments that demonstrate some of the constraints of unitarity on four-particle scattering amplitudes. If the scattering particles have equal masses, $\mu$, their momenta $k_{r}$ $(r=1,2,3,4)$, are $D$-dimensional Minkowski vectors satisfying the mass-shell conditions $k_{r}$. $k_{r}=-\mu^{2}$. Such amplitudes are functions of the Mandelstam invariants $s_{i j}=-\left(k_{i}+k_{j}\right)^{2}=$ $2 \mu^{2}-2 k_{i} \cdot k_{j}$. As usual we will use the notation $s_{12}=s_{34}=s, s_{14}=s_{23}=t, s_{13}=s_{24}=u$, and recall that momentum conservation $\sum_{r} k_{r}=0$ implies $s+t+u=4 \mu^{2}$. In addition, the external particles generally have non-zero spin and so the scattering data includes information about their polarisations, although this will be suppressed in most of the following.

The amplitude $A_{4}(s, t)$ is an analytic function of $s, t$ and $u$, apart from a very specific set of singularities. The physical region for the elastic scattering process $1+2 \rightarrow 3+4$ with centre of mass energy $2 E=\sqrt{s}$ and scattering angle $\cos \theta=1+2 t /\left(s-4 \mu^{2}\right)$ is $s \geq 4 \mu^{2}, 4 \mu^{2}-s \leq t \leq 0,4 \mu^{2}-s \leq u \leq 0$. The amplitudes for other physical regions in which $1+4 \rightarrow 2+3$ and $1+3 \rightarrow 2+4$ are related by appropriate analytic continuation (or crossing symmetry). The singularities of the amplitude include poles corresponding to intermediate bound states or resonances, normal threshold branch cuts corresponding to the production of intermediate multi-particle states, and anomalous thresholds that lie outside the physical scattering region. ${ }^{4}$

We will be concerned with theories that exhibit "duality", such as string theory, or the large- $N$ expansion of SU $(N)$ Yang-Mills theory. The tree-level contribution to four-particle scattering amplitudes in such theories (i.e. the leading perturbative contributions in string perturbation theory, or in the $1 / N$ expansion in $\mathrm{SU}(N)$ Yang-Mills theory) necessarily possess an infinite sequence of poles at positions $m_{a}^{2}(a=1, \ldots, \infty)$ along the positive real $s, t$ and $u$ axes. In the full non-perturbative amplitude unitarity implies the presence of branch cuts along the real $s, t$ and $u$ axes and almost all the poles are shifted below the real $s, t$ or $u$ axes and are shielded from the physical sheet behind branch cuts.

A general feature of unitarity that plays a key rôle in the following is the optical theorem, which states

$$
\left.\operatorname{Im} A_{4}(s, 0)=\sqrt{s\left(s-4 \mu^{2}\right.}\right) \sigma_{\text {tot }}(s)>0,
$$

\footnotetext{
${ }^{3} \mathrm{An}$ irreducible MZV is one that cannot be expressed as a rational polynomial in single zeta values.

${ }^{4} \mathrm{~A}$ basic review of the singularity structure of the S-matrix is given in [25]. However, this is restricted to the amplitudes with massive scattering particles with zero angular momenta.
} 
where $\sigma_{\text {tot }}$ is the total cross section, which is positive. A stronger set of positivity conditions of the form

$$
\left.\partial_{t}^{n} \operatorname{Im} A(s, t)\right|_{t=0}>0, \quad \forall n>0
$$

can be deduced from properties of the partial wave expansion.

In this paper we will restrict attention to tree-level expressions in dual resonance theories. In such cases a scalar amplitude can be written in the $s$-channel partial wave form ${ }^{5}$

$$
A_{4}(s, t)=A_{4}^{(s)}(s, t)+A_{4}^{(u)}(u, t),
$$

where

$$
A_{4}^{(s)}(s, t)=\sum_{a} \frac{p_{a} G_{\ell_{a}}^{\frac{D-3}{2}}\left(\cos \theta_{a}\right)}{m_{a}^{2}-s}, \quad A_{4}^{(u)}(u, t)=\sum_{a} \frac{p_{a} G_{\ell_{a}}^{\frac{D-3}{2}}\left(\cos \theta_{a}\right)}{m_{a}^{2}-u}
$$

and

$$
\cos \theta_{a}=1+\frac{2 t}{m_{a}^{2}}
$$

These expressions are sums of poles describing intermediate states with masses $m_{a}$ and angular momenta $\ell_{a}$ in the $s$ and $u$ channels. The residue of a given pole of angiular momentum $\ell$ is proportional to the Gegenbauer polynomial $G_{\ell}^{\frac{D-3}{2}}\left(\cos \theta_{a}\right.$ ) (which is equal to the Legendre polynomial $P_{\ell}\left(\cos \theta_{a}\right)$ when $\left.D=4\right) .{ }^{6}$ The $s$-channel and $u$-channel centre of mass scattering angles are evaluated at the poles, so $\cos \theta_{a}=1+2 t / m_{a}^{2}$. The proportionality constants $p_{a}$ denote the squares of the coupling between a pair of external scalar states and the intermediate states of masses $m_{a}$ and angular momenta $\ell_{a}$, and so they are positive for a unitary theory. Sincer $\left.\partial_{y}^{n} G_{\ell}^{\frac{D-3}{2}}(y)\right|_{y=1}>0$ for all $n$, the positivity conditions (2.2) are satisfied if the couplings satisfy $p_{a}>0$ for all $a$.

The expression (2.4) is appropriate for describing the physical amplitude $1+2 \rightarrow 3+4$ with $s>0$ and fixed $-s \leq t \leq 0$. However, it is defined for all values of $s, t, u$ (with $s+t+u=0$ ) by analytic continuation. The presence of poles at $t \geq 0$ that contribute to the crossed process $1+4 \rightarrow 2+3$ requires the index $a$ in (2.4) to take an infinite number of values so there is an infinite number of $s$-channel and/or $u$-channel poles with unbounded masses, $m_{a} \underset{a \rightarrow \infty}{\rightarrow} \infty$. Indeed, the requirement of an infinite number of tree-level poles is the hallmark of dual resonance models and the gauge-singlet sectors of large- $N$ QCD.

The structure of $A_{4}(s, t)$ differs from that of a tree contribution to a conventional quantum field theory with a finite number of fields, in which there would be explicit pole contributions in the $t$ channel, as well as in the $s$, and $u$ channels. Adding $t$-channel poles would lead to an additional polynomial in $s$ in (2.3), which would markedly affect its large- $|s|$ behaviour.

\footnotetext{
${ }^{5}$ We are imposing the mass-shell condition $u=-s-t$ appropriate for scattering of massless states, in the following.

${ }^{6}$ A generating function for Gegenbauer polynomials may be defined by

$$
\frac{1}{\left(1-2 x t+t^{2}\right)^{m}}=\sum_{\ell=0}^{\infty} G_{\ell}^{(m)}(x) t^{\ell}
$$


More generally, the external states may have spin and this will be reflected by spindependent factors that complicate these expressions. For the purpose of this paper, we will focus on the amplitudes where external states are scalars, This is sufficient for our later considerations of maximally supersymmetric superstring amplitudes since in such theories a supermultiplet of massless states is described in terms of a Lorentz-scalar on-shell superfield.

An important technical point is that the amplitude is generally singular at the boundaries of the physical region when $s>0$ and $t=0$ or $t=-s$, i.e. when $\theta=0$ or $\theta=\pi$. In Yang-Mills theory and gravity this is due to the exchange of a massless gauge boson or graviton. In order to discuss the low-energy expansion of the amplitude it is therefore important to subtract the singular term, which we denote $A_{\text {sing }}(s, t)$. Thus we may define a subtracted amplitude

$$
\tilde{A}_{4}(s, t)=A_{4}(s, t)-A_{\text {sing }}(s, t),
$$

which is finite in the forward and backward limits, $t=0$ and $t=-s$.

The low energy expansion of $\tilde{A}_{4}(s, t)$ in powers of $s$ and $t$ is given by

$$
\left.\tilde{A}_{4}(s, t)\right|_{s / m^{2}, t / m^{2} \ll 1}=\sum_{p, q=0} g_{p, q} s^{p} t^{q}
$$

The coefficients in this expansion are related to ultraviolet physics by virtue of the analyticity of the S-matrix by considering the Cauchy integral around a small circle enclosing the point $s=0$. For a fixed $t$,

$$
\begin{aligned}
\sum_{q} g_{p, q} t^{q}= & \frac{1}{2 \pi i} \oint \frac{d s}{s^{p+1}} \tilde{A}_{4}(s, t) \\
= & \frac{1}{\pi} \int_{s=0}^{\infty} \frac{d s}{s^{p+1}} \operatorname{Im} \tilde{A}_{4}^{(s)}(s, t)+\frac{1}{\pi} \int_{s=-t}^{\infty} \frac{d s}{s^{p+1}} \operatorname{Im} \tilde{A}_{4}^{(u)}(-s-t, t) \\
& +\left.\frac{1}{2 \pi i} \int_{0}^{2 \pi} d \varphi \frac{d s}{s^{p+1}} \tilde{A}_{4}(s, t)\right|_{|s| \rightarrow \infty},
\end{aligned}
$$

where the integration contour in the first line is a circle around the origin. In passing to the second line the contour has been deformed to pick up the contribution from the discontinuity $\operatorname{Disc} A(s, t)=\lim _{\epsilon \rightarrow 0}(A(s+i \epsilon, t)-A(s-i \epsilon))=2 i \operatorname{Im} \tilde{A}(s, t)$. If the subtracted amplitude behaves as $\tilde{A}_{4}(s, t)<|s|^{w}$ as $s \rightarrow \infty$ the contribution from the contour at infinity (the third line in (2.9)) vanishes for all $p \geq w$ (where we have defined $s=|s| e^{i \varphi}$ ). From hereon we will assume that $w<0$ so this contribution can be dropped. This will be shown to be true in the explicit examples that we will consider later although it is not true in most conventional field theory examples.

In the tree-level examples of the form $(2.3) \operatorname{Im} \tilde{A}_{4}^{(s)}(s, t)$ is a sum of delta functions of the form ${ }^{7}$

$$
\operatorname{Im} \tilde{A}_{4}^{(s)}(s, t)=\pi \sum_{a} p_{a} G_{\ell_{a}}^{\frac{D-3}{2}}\left(1+\frac{2 t}{m_{a}^{2}}\right) \delta\left(s-m_{a}^{2}\right),
$$

\footnotetext{
${ }^{7}$ Note that the discontinuity of a pole at the origin in the complex $z$-plane is given by $\operatorname{Disc} 1 / z=$ $-2 \pi i \delta(|z|)$.
} 
and so the contribution from the $s$-channel poles in (2.9) is given by

$$
\sum_{q} g_{p, q}^{(s)} t^{q}=\frac{1}{\pi} \int_{s=0}^{\infty} \frac{d s}{s^{p+1}} \operatorname{Im} \tilde{A}_{4}^{(s)}(s, t)=\sum_{a} \frac{p_{a}}{m_{a}^{2 p+2}} G_{\ell_{a}}^{\frac{D-2}{2}}\left(1+\frac{2 t}{m_{a}^{2}}\right) .
$$

It is important to note that (2.10) implies that $\left.\partial_{t}^{n} \operatorname{Im} \tilde{A}_{4}^{(s)}(s, t)\right|_{t=0}>0$.

By contrast, the contribution of the $u$-channel poles is

$$
\sum_{q} g_{p, q}^{(u)} t^{q}=\frac{1}{\pi} \int_{s=-t}^{-\infty} \frac{d s}{s^{p+1}} \operatorname{Im} \tilde{A}_{4}^{(u)}(s, t)=\sum_{a} \frac{(-1)^{p} p_{a}}{\left(t+m_{a}^{2}\right)^{p+1}} G_{\ell_{a}}^{\frac{D-2}{2}}\left(1+\frac{2 t}{m_{a}^{2}}\right) .
$$

It follows that $\left.\partial_{t}^{n} \operatorname{Im} \tilde{A}_{4}^{(u))}(s, t)\right|_{t=0}$ has indefinite sign as do the coefficients $g_{p, q}^{(u)}$. The full coefficient of $s^{p} t^{q}$ is given by $g_{p, q}=g_{p, q}^{(s)}+g_{p, q}^{(u)}$ and therefore in general it has indefinite sign when there are $u$-channel poles, but in special cases $g_{p, q}$ is positive.

\subsection{The Stieltjes half-moment sequence}

It is useful to consider the $n \times n$ Hankel matrices composed of the expansion coefficients $g_{p, q}$. Thus, we may define a $(n+1) \times(n+1)$ Hankel matrix, which depends on $n+1$ sequential coefficients for a given value of $q, \mathbf{g}_{\mathbf{q}}=\left\{g_{m, q}, g_{m+1, q} \ldots, g_{m+n, q}\right\}$

$$
H_{n}^{(m)}\left[\mathbf{g}_{\mathbf{q}}\right]=\left(\begin{array}{cccc}
g_{m, q} & g_{m+1, q} & \cdots & g_{m+n, q} \\
g_{m+1, q} & g_{m+2, q} & \cdots & g_{m+n+1, q} \\
\vdots & \vdots & \vdots & \vdots \\
g_{m+n, q} & g_{m+n+1, q} & \cdots & g_{m+2 n, q}
\end{array}\right) .
$$

The following theorem concerning positivity conditions on Hankel matrices (an abbreviated version of Theorem 2.8 in [19]) is of central importance in the following:

Given a sequence of real coefficients, $\mathbf{a}=\left(a_{0}, a_{1}, \ldots, a_{\infty}\right)$ then the following statements are equivalent.

- The infinite dimension Hankel matrix of the coefficients, $H_{\infty}^{(0)}(\mathbf{a})$ is totally positive, so all its $n \times n$ minors are positive for all $n$.

- The leading principal minors $\operatorname{det} H_{n}^{(0)}(\mathbf{a})$ and $\operatorname{det} H_{n}^{(1)}(\mathbf{a})$ are positive definite for all $n$.

- There is a positive measure $\mu$ on $[0, \infty)$ whose support is the infinite set

$$
a_{k}=\int_{0}^{\infty} y^{k} d \mu(y), \quad \forall k \geq 0 .
$$

This means that $\left(a_{0}, a_{1}, \ldots, a_{\infty}\right)$ form a Stieltjes half-moment sequence.

This theorem is relevant if, for example, we make the identifications $a_{p} \rightarrow g_{p, q}, y \rightarrow 1 / s$ and $\left.d \mu(y) \rightarrow \partial_{t}^{q} \operatorname{Im} A^{(s)}(s, t)\right|_{t=0} d s / s$, where $\operatorname{Im} A^{(s)}(s, t)$ is given in (2.10). 


\subsection{Positivity conditions on moments of the amplitude}

We will now consider the first few terms in the expansion of $\tilde{A}_{4}(s, t)$ in powers of $t$, which leads to expressions for the individual entries of the matrix $g_{p, q}$.

Terms of order $t^{0}$. The terms of lowest order in $t$ are the coefficients of $t^{0}$, which form the vector $g_{p, 0}$ that is associated with the forward limit $t=0$ in (2.9). In this case we have $g_{p, 0}^{(s)}=g_{p, 0}^{(u)}$, which results in

$$
g_{p, 0}=\sum_{a} \frac{2 p_{a}}{m_{a}^{2 p+2}} G_{\ell_{a}}^{\frac{D-2}{2}}(1)
$$

which is equivalent to

$$
\left(\begin{array}{c}
g_{0,0} \\
g_{1,0} \\
g_{2,0} \\
\vdots
\end{array}\right)=\sum_{a} \frac{2 p_{a}}{m_{a}^{2}} G_{\ell_{a}}^{\frac{D-2}{2}}(1)\left(\begin{array}{c}
x_{a} \\
x_{a}^{2} \\
x_{a}^{3} \\
\vdots
\end{array}\right)
$$

where $x_{a}=m_{a}^{-2} \in R^{+}$. The set of coefficients, $\mathbf{g}_{0}=\left\{g_{0,0}, g_{1,0} \ldots, g_{n, 0}\right\}$ (for any $n$ ) defines a $(n+1) \times(n+1)$ Hankel matrix

$$
H_{n}^{(0)}\left[\mathbf{g}_{\mathbf{0}}\right]=\left(\begin{array}{ccccc}
g_{0,0} & g_{1,0} & g_{2,0} & \cdots & g_{n, 0} \\
g_{1,0} & g_{2,0} & g_{3,0} & \ldots & g_{n+1,0} \\
g_{2,0} & g_{3,0} & g_{4,0} & \cdots & g_{n+2,0} \\
\vdots & \vdots & \vdots & \vdots & \vdots \\
g_{n, 0} & g_{n+1,0} & g_{n+2,0} & \cdots & g_{2 n, 0}
\end{array}\right)
$$

In this case we identify $a_{p}=g_{p, 0}$ in (2.14) and the measure has the form $\sum_{a} 2 p_{a} G_{\ell_{a}}^{\frac{D-2}{2}}(1) / m_{a}^{2} \delta\left(y-x_{a}\right) d y$. This is equivalent to the statement that the coefficients $g_{p, 0}$ in (2.16) reside in the convex hull of points of a half moment curve with components $x_{a}^{n}$. According to the theorem stated above this property implies that the Hankel matrices built out of the coefficients $g_{m, 0}$ are totally positive so all the minors of the Hankel matrix are positive definite. ${ }^{8}$

Using the expressions for $g_{m, 0}$ in $(2.16)$ it is straightforward to show explicitly that

$$
\operatorname{det} H_{n}^{(0)}\left[\mathbf{g}_{0}\right]=\sum_{P} \prod_{i \in P} \frac{p_{i}}{m_{i}^{2}} \prod_{i<j \in P}\left(x_{i}-x_{j}\right)^{2} \geq 0,
$$

where $P$ is any length $n$ subset of $\{1,2, \ldots, L\}$, with $L$ being the upper limit of the sum in (2.16). If $L$ is finite then it follows that $\operatorname{det} H_{n}^{(0)}\left[\mathbf{g}_{\mathbf{0}}\right]=0$ for $n>L$, and $H_{n}^{(0)}\left[\mathbf{g}_{0}\right]$ is non-negative, i.e, $\operatorname{det} H_{n}^{(0)}\left[\mathbf{g}_{\mathbf{0}}\right] \geq 0$. In theories such as string theory or large- $N$ QCD the

\footnotetext{
${ }^{8}$ Notational comments: a minor of a $n \times n$ matrix is the determinant of a $k \times k$ sub-matrix with $k<n$ obtained by deleting $n-k$ rows and $n-k$ columns. A principal minor is the determinant of a $k \times k$ submatrix obtained by deleting $n-k$ columns and the $n-k$ rows that have the same numbering. A leading principal minor is the determinant of a sub-matrix obtained by deleting the last $n-k$ columns and rows.
} 
number of tree-level poles is infinite $(L=\infty)$ and $H_{\infty}^{(0)}\left[\mathbf{g}_{0}\right]$ is totally positive. Analogously, one can show the positivity of $\operatorname{det} H_{n}^{(0)}\left[\mathbf{g}_{0}\right]$ with $\mathbf{g}_{0}=\left\{g_{m, 0}, g_{m+1,0} \ldots, g_{m+n, 0}\right\}$. The result is simply given by (2.18) with $\frac{p_{i}}{m_{i}^{2}} \rightarrow \frac{p_{i}}{m_{i}^{2}} x_{i}^{m}$. As stated previously, the positivity of $\operatorname{det} H_{n}^{(0)}\left[\mathbf{g}_{\mathbf{0}}\right]$ and $\operatorname{det} H_{n}^{(1)}\left[\mathbf{g}_{\mathbf{0}}\right]$ is equivalent to the fact that the Hankel matrix $H_{\infty}^{(0)}\left[\mathbf{g}_{\mathbf{0}}\right]$ is totally positive.

Terms of higher order in $\boldsymbol{t}$. In order to consider the low energy expansion of tree amplitudes to higher powers of $t$ we first expand the propagators in (2.3) and (2.4) to all orders in $s$, giving

$$
\begin{aligned}
& A_{4}^{(s)}(s, t)=\sum_{a} \frac{p_{a}}{m_{a}^{2}} G_{\ell_{a}}^{\frac{D-3}{2}}\left(1+\frac{2 t}{m_{a}^{2}}\right) \sum_{n=0}^{\infty} \frac{s^{n}}{m_{a}^{2 n}}, \\
& A_{4}^{(u)}(s, t)=\sum_{a} \frac{p_{a}}{m_{a}^{2}} G_{\ell_{a}}^{\frac{D-3}{2}}\left(1+\frac{2 t}{m_{a}^{2}}\right) \sum_{n=0}^{\infty} \frac{(-s-t)^{n}}{m_{a}^{2 n}} .
\end{aligned}
$$

Notice that the terms of order $s^{2 n+1} t^{0}$ cancel in the sum of $A_{4}^{(s)}(s, t)$ and $A_{4}^{(u)}(s, t)$, whereas the terms of order $s^{2 n} t^{0}$ double, giving the positive definite coefficients discussed earlier. However, the expansion of $A_{4}^{(u)}(s, t)$ in (2.20) in powers of $s$ for a fixed power $t^{q}$ with $q>0$ has negative contributions that do not cancel with the terms in $A_{4}^{(s)}(s, t)$. Therefore the positivity condition for $A_{4}(s, t)$ is more involved, although more subtle positivity statements can still be obtained as in [11-13] and [14, 15]. For our purposes it will be sufficient to consider properties of the low-energy expansions of $A_{4}^{(s)}(s, t)$ and $A_{4}^{(u)}(s, t)$ contributions separately.

In the case of open-string amplitudes or large- $N$ QCD meson amplitudes the building blocks (such as the colour-stripped amplitudes) only have $s$-channel poles (or $u$-channel poles) for a fixed value of $t$. In such cases only $A_{4}^{(s)}(s, t)$ (or $A_{4}^{(u)}(u, t)$ ) contributes to the low-energy expansion, and has an expansion of the form

$$
A_{4}^{(s)}(s, t)=\left.\sum_{p, q=0}^{\infty} \sum_{a} p_{a} \frac{2^{q}}{q ! m_{a}^{2}} \partial_{y}^{q} G_{\ell_{a}}^{\frac{D-3}{2}}(y)\right|_{y=1}\left(\frac{s^{p}}{m_{a}^{2 p}}\right)\left(\frac{t^{q}}{m_{a}^{2 q}}\right) .
$$

Noting the property of Gegenbauer polynomials $\left.\partial_{y}^{q} G_{\ell_{a}}^{\frac{D-3}{2}}(y)\right|_{y=1}>0$ and recalling that $p_{a}>0$ the coefficients in (2.21) satisfy

$$
\left.\frac{2^{q} p_{a}}{m_{a}^{2+2 q}} \partial_{y}^{q} G_{\ell, a}^{\frac{D-3}{2}}(y)\right|_{y=1}>0
$$

So we see that, at any given order $t^{q}$, the low-energy expansion again defines a half moment curve, so the Hankel matrices formed by the low-energy coefficients are totally non-negative.

More generally, amplitudes have both $s$-channel and $u$-channel poles so both $A_{4}^{(s)}(s, t)$ and $A_{4}^{(u)}(s, t)$ contribute. In such cases the coefficients in the low energy expansion are not necessarily positive and do not reside on a moment curve (apart from the special $t^{0}$ case) and there is no straightforward condition on the Hankel matrices. However the coefficients of the low-energy expansion of the term with $s$-channel poles, $A_{4}^{(s)}(s, t)$ do satisfy positivity 
conditions that again generally lead to totally non-negative Hankel matrices. As before, when the range of $a$ is infinite (as is the case with closed-string amplitudes and glueball amplitudes in large- $N$ QCD) the Hankel matrices are totally positive. The term with $u$ channel poles, $A_{4}^{(u)}(u, t)$, satisfies the same conditions when expanded in powers of $u$ for a fixed power of $t$. We will see that in the closed-string case this leads to totally positive Hankel matrix determinants with entries that are rational linear combinations of MZVs.

\section{$3 \quad$ Four-particle open superstring tree amplitudes}

In the rest of this paper we will consider critical superstring theory amplitudes, which have massless sectors describing maximally supersymmetric Yang-Mills theory in the case of open strings and maximally supersymmetric gravity in the case of closed strings.

After stripping off the colour factors the amplitude that describes the scattering of any four massless particles in the Yang-Mills supermultiplet has a term of the form

$$
\mathcal{A}_{\text {op }}(s, t)=P_{4} A_{o p}(s, t),
$$

that contains poles in the $s$ and $t$ channels. The factor $P_{4}$ is a dimension-four kinematic prefactor that is determined by maximal Yang-Mills supersymmetry and contains the information about which particular states are being scattered. For example, in the case of the fourgluon amplitude this prefactor is given by $P_{4}=F^{4}$, where $F$ is the linearised field strength. ${ }^{9}$

The amplitude (3.1) contains the stringy corrections to the field theory tree-level amplitude, which is $P_{4} /(s t)$. In order to obtain an expression that is well-defined at $t=0$, we will again consider the amplitude after subtraction of the super Yang-Mills tree-level term,

$$
\begin{aligned}
\tilde{A}_{o p}(s, t):=A_{o p}(s, t)+\frac{1}{s t} & =-\frac{1}{s t}\left(\frac{\Gamma(1-s) \Gamma(1-t)}{\Gamma(1-(s+t))}-1\right) \\
& =-\frac{1}{s t}\left(\exp \left[\sum_{k=2}^{\infty} \frac{\zeta(k)}{k}\left(s^{k}+t^{k}-(s+t)^{k}\right)\right]-1\right) .
\end{aligned}
$$

From the expression in the second line it is obvious that $\tilde{A}_{o p}(s, t)$ has a low energy expansion in powers of $s$ and $t$ with coefficients that are rational polynomials in Riemann zeta values. The amplitude $\tilde{A}_{o p}(s, t)$ can also be written as a sum of $s$-channel poles by using the integral representation for the Euler beta function,

$$
\begin{aligned}
\tilde{A}_{o p}(s, t) & =\frac{1}{t} \int_{0}^{1} d x x^{-1-s}(1-x)^{-t}+\frac{1}{s t} \\
& =\frac{1}{t} \int_{0}^{1} \frac{d x}{x} \sum_{m=1}^{\infty} x^{-s+m}(-1)^{m}\left(\begin{array}{c}
-t \\
m
\end{array}\right) \\
& =\sum_{m=1}^{\infty} \frac{1}{m-s} \frac{\Gamma(m+t)}{\Gamma(1+t) \Gamma(1+m)}=\sum_{m=1}^{\infty} \sum_{\ell_{m}=0}^{m-1} \frac{\left.p_{m}^{\ell_{m}} G_{\ell_{m}}^{\frac{D-3}{2}}\left(1+\frac{2 t}{m}\right)\right)}{m-s} .
\end{aligned}
$$

\footnotetext{
${ }^{9}$ The manifestly supersymmetric amplitude from which the component expression (3.1) arises can be expressed as $\delta^{8}\left(Q_{4}\right) A_{o p}(s, t)$, where $Q_{4}$ is the supercharge for the four scattering states.
} 
Writing the amplitude in this manner exhibits the infinite set of poles at positive integer values of $s$, but obscures the $s-t$ symmetry of the amplitude and, in particular, obscures the presence of an infinite set of poles at positive integer values of $t$. The last equality of (3.3) expresses the amplitude in the form of a partial wave sum over Gegenbauer polynomials of the same form as $A_{4}^{(s)}(s, t)$ in $(2.4)$ (where $m_{a}^{2}$ takes integer values, $m$, and the angular momentum of states at mass $m$ takes the values, $\left.0 \leq \ell_{m} \leq m+1\right)$. The identity in the last line only implies $p_{m}^{\ell_{m}} \geq 0$ when $D \leq 10$, which is consistent with the no-ghost theorem. A more complete derivation of the amplitude (3.2) requires the condition $D=10$ of the critical superstring.

The asymptotic behaviour of $\tilde{A}_{o p}(s, t)$ as $|s| \rightarrow \infty$ with $\epsilon<\arg (s)<2 \pi-\epsilon$, and $t \leq 0$, can be obtained by using Stirling's approximation, giving

$$
\tilde{A}_{o p}(s, t) \underset{s \rightarrow \infty}{\sim} \frac{1}{s t}-(-s)^{t-1}\left(\Gamma(-t)+O\left(s^{-1}\right)\right),
$$

so the last term in (2.9) (the large-s contour integral) can be dropped.

\subsection{Low-energy expansion of the four-particle open superstring tree amplitude}

The expansion of the expression (3.3) in powers of $s$ and $t$ is straightforward and has the form

$$
\tilde{A}_{o p}(s, t)=\sum_{p, q=0}^{\infty} g_{p, q}^{o p} s^{p} t^{q} .
$$

The coefficients in this expansion, $g_{p, q}^{o p}$, are rational polynomials in Riemann zeta values with weights $w=p+q+2$. The terms up to order $t^{3}$ and order $s^{5}$ are shown in (A.1).

The $(p, 0)$ terms. The leading power of $t$ is picked out by considering the forward limit, $t=0$, in which case the amplitude reduces to the simple form

$$
\begin{aligned}
\tilde{A}_{o p}(s, 0) & =\sum_{n=0}^{\infty} \zeta(n+2) s^{n} \\
& =-\frac{1}{s}(\gamma+\psi(1-s)) \\
& =\sum_{n=1}^{\infty} \frac{1}{n(n-s)}=\frac{1}{s} \sum_{n=1}^{\infty}\left(\frac{1}{n-s}-\frac{1}{n}\right),
\end{aligned}
$$

where the digamma function is defined by $\psi(z)=\Gamma^{\prime}(z) / \Gamma(z)$ and the Euler-Mascharoni constant is defined by $\gamma=-\psi(1)=-\Gamma^{\prime}(1)$. It follows that in this case the coefficients, $g_{p, 0}^{o p}$ are simply given by

$$
g_{p, 0}^{o p}=\zeta(p+2) .
$$

We also see from (3.6) that the $t=0$ contribution may be expressed as an infinite sum of poles with positive residues in accord with unitarity. It follows from our previous discussion that the determinants of the $n \times n$ Hankel matrices with $\zeta(i+j)$ entries (where $i$ is even or odd) are all positive, as are all the minors of these matrices. 
Such determinants of Hankel matrices with $\zeta$-value entries have been considered in the mathematics literature $[23,24]$. These references considered the behaviour of the determinants of the $2 n \times 2 n$ Hankel matrices

$$
\operatorname{det} H_{n}^{(0)}[\zeta]_{i j}=\operatorname{det}(\zeta(i+j)), \quad i, j=1,2, \ldots, n,
$$

and

$$
\operatorname{det} H_{n}^{(1)}[\zeta]_{i j}=\operatorname{det}(\zeta(i+j+1)), \quad i, j=1,2, \ldots, n,
$$

which were both argued to be positive. This follows from the fact that $\operatorname{det} H_{n}^{(1)}[\zeta]_{i j}$ is a principal minor of $H_{n+1}^{(0)}[\zeta]_{i j}$. In fact, as we commented previously, it is a property of the Stieltjes moment coefficients that if two such Hankel matrices are known to have positive determinants, all other minors are positive [19].

It is easy to see that $\operatorname{det} H_{n}^{(i)}[\zeta](i=0,1)$ approaches zero rapidly as $n$ increases. More explicitly, it was reported in $[23,24]$ that the asymptotic values of these determinants at large $n$ are given by the expressions ${ }^{10}$

$$
\operatorname{det} H_{n}^{(0)}[\zeta]=d^{(0)}\left(\frac{2 n+1}{e \sqrt{e}}\right)^{-(n+1 / 2)^{2}}\left(1+\frac{1}{24} \frac{1}{(2 n+1)^{2}}+\ldots\right),
$$

and

$$
\operatorname{det} H_{n-1}^{(1)}[\zeta]=\frac{e^{9 / 8}}{\sqrt{6}} d^{(0)}\left(\frac{2 n}{e \sqrt{e}}\right)^{-n^{2}+3 / 4}\left(1-\frac{17}{240} \frac{1}{(2 n)^{2}}+\ldots\right),
$$

It is easy to check these expressions with help from Mathematica, although we find the numerical constant $d^{(0)}=0.66367$ rather than the value attributed to Zagier in [23], which is $d^{(0)}=0.35147$.

The $(p, \mathbf{1})$ terms. It is also easy to see that the $q=1$ terms (terms of order $t^{1}$ ) are given by

$$
\left.\partial_{t} \tilde{A}_{o p}^{(1)}(s, t)\right|_{t=0}=\sum_{p=0}^{\infty} g_{p, 1}^{o p} s^{p}
$$

with

$$
g_{p, 1}^{o p}=\frac{p+2}{2} \zeta(p+3)-\frac{1}{2} \sum_{i=1}^{p} \zeta(i+1) \zeta(p+2-i) .
$$

In this case one may construct Hankel matrices $H_{n}^{o p(0)}\left[\mathbf{g}_{\mathbf{1}}^{\mathbf{o p}}\right]$ of the form $(2.17)$ with entries $\left\{g_{0,1}^{o p}, \ldots, g_{n, 1}^{o p}\right\}$ in the first row. The determinants of such matrices and all their minors again satisfy positivity conditions. Since $g_{p, 1}^{o p}$ is quadratic in zeta values such bounds now imply more complicated bounds on rational polynomials of zeta values.

\footnotetext{
${ }^{10}$ The constants $d^{(0)}$ was denoted $A^{(0)}$ in [23].
} 
All $(\boldsymbol{p}, \boldsymbol{q})$ terms. It is tedious to extract the complete set of coefficients for $q>1$ simply by expanding the expression in (3.2). However, it was shown recently [26] (see also [27]) that the coefficient $g_{p, q}^{o p}$ is given by the special multiple zeta values ${ }^{11}$ (which can also be expressed as special kinds of Mordell-Tornheim sums)

$$
g_{p, q}^{o p}=\zeta(\underbrace{1, \cdots, 1}_{q}, p+2) .
$$

These expressions can be reduced to rational polynomials in single zeta values of total weight $p+q+2$ by comparing the coefficient of $s^{p} t^{q}$ in the low energy expansion of the open-string amplitude (3.2) with (3.14). In this manner we have been able to determine the following expressions for the coefficients with $q=0, \ldots, 3$ for all $p \geq 0$,

$$
\begin{aligned}
& q=0: \quad \quad \zeta(p+2), \\
& q=1: \quad \zeta(1, p+2)=\frac{p+2}{2} \zeta(p+3)-\frac{1}{2} \sum_{i=1}^{p} \zeta(i+1) \zeta(p+2-i), \\
& q=2: \quad \zeta(1,1, p+2)=\frac{(p+2)(p+3)}{3 !} \zeta(p+4)-\sum_{i=1}^{p} \frac{i+1}{2 !} \zeta(i+2) \zeta(p+2-i) \\
& +\frac{1}{3 !} \sum_{\substack{i, j=1 \\
i+j \leq p}}^{p} \zeta(i+1) \zeta(j+1) \zeta(p+2-i-j), \\
& q=3: \quad \zeta(1,1,1, p+2)=\frac{(p+2)(p+3)(p+4)}{4 !} \zeta(p+5) \\
& -\sum_{i=1}^{p} \frac{(i+1)(i+2)}{3 !} \zeta(i+3) \zeta(p+2-i) \\
& -\frac{1}{2 !} \sum_{i=1}^{p} \frac{i+1}{2 !} \frac{p+2-i}{2 !} \zeta(i+2) \zeta(p+3-i) \\
& +\frac{1}{2 !} \sum_{\substack{i, j=1 \\
i+j \leq p}}^{p} \frac{j+1}{2 !} \zeta(i+1) \zeta(j+2) \zeta(p+2-i-j) \\
& -\frac{1}{4 !} \sum_{\substack{i, j, k=1 \\
i+j+k \leq p}}^{p} \zeta(i+1) \zeta(j+1) \zeta(k+1) \zeta(p+2-i-j-k) .
\end{aligned}
$$

The class of $n \times n$ Hankel matrices that is generated from the low-energy expansion of the open-string four-particle amplitudes at a given order $t^{q}$ is given by

$$
H_{n}^{o p}\left[\zeta_{\mathbf{q}}\right]_{i j}=\zeta(\underbrace{1, \cdots, 1}_{q}, i+j), \quad i, j=1,2, \ldots, n,
$$

where the matrices with $n=1,2,3, \cdots$. are sub-matrices of the infinite-dimensional matrix $H_{\infty}^{o p}[\zeta]_{i j}$. The notation $H_{n}^{o p}\left[\zeta_{\mathbf{q}}\right]$ denotes the Hankel matrix with the first row defined by

\footnotetext{
${ }^{11} \mathrm{~A}$ general multiple zeta value of depth $r$ and weight $w=\sum_{i=1}^{r} k_{i}$ is defined by $\zeta\left(k_{1}, k_{2}, \ldots, k_{r}\right)=$ $\sum_{0<n_{1}<\cdots<n_{r}} n_{1}^{-k_{1}} \ldots n_{r}^{-k_{r}}$.
} 
the sequence $\{\zeta(\underbrace{1, \cdots, 1}_{q}, 2), \ldots, \zeta(\underbrace{1, \cdots, 1}_{q}, n+1)\}$. For example, the Hankel matrices with $n=3$ and general $q$ have the form

$$
H_{3}^{o p}\left[\zeta_{\mathbf{q}}\right]=\left(\begin{array}{l}
\zeta(\underbrace{1, \cdots, 1}_{q}, 2) \zeta(\underbrace{1, \cdots, 1}_{q}, 3) \zeta(\underbrace{1, \cdots, 1}_{q}, 4) \\
\zeta(\underbrace{1, \cdots, 1}_{q}, 3) \zeta(\underbrace{1, \cdots, 1}_{q}, 4) \zeta(\underbrace{1, \cdots, 1}_{q}, 5) \\
\zeta(\underbrace{1, \cdots, 1}_{q}, 4) \zeta(\underbrace{1, \cdots, 1}_{q}, 5) \zeta(\underbrace{1, \cdots, 1}_{q}, 6)
\end{array}\right) .
$$

The unitarity constraints again imply that

$$
\operatorname{det} H_{n}^{o p}\left[\zeta_{\mathbf{q}}\right]>0
$$

for all $n \geq 1$ and $q \geq 0$, as well as similar positivity constraints on all the minors. The simplest example of many such constraints comes from the positivity of the upper left $2 \times 2$ minor

$$
\zeta(\underbrace{1, \cdots, 1}_{q}, 2) \zeta(\underbrace{1, \cdots, 1}_{q}, 4)-(\zeta(\underbrace{1, \cdots, 1}_{q}, 3))^{2}>0 .
$$

This is just one of an infinite number of positivity constraints that can be reduced to inequalities on polynomials of positive zeta values. It is straightforward to check these numerically and to check that $\operatorname{det} H_{n}^{o p}\left[\zeta_{\mathbf{q}}\right]$ decreases rapidly to zero as $n$ grows. However, we have not obtained expressions analogous to (3.10) and (3.11), which would give the asymptotic dependence of $\operatorname{det} H_{n}^{o p}\left[\zeta_{\mathbf{q}}\right]$ on $n$.

\section{Four-particle closed superstring tree amplitudes}

The four-particle closed-string tree amplitude has the form

$$
\mathcal{A}_{c l}(s, t)=P_{8} A_{c l}(s, t)
$$

where $P_{8}$ is a dimension-eight kinematic factor that is determined by supersymmetry, such as $R^{4}$ in the case of the four-graviton amplitude (where $R$ is the linearised Riemann curvature). ${ }^{12}$ The factor $A_{c l}(s, t)$ is given by

$$
\begin{aligned}
A_{c l}(s, t) & =-\frac{1}{s t(s+t)} \frac{\Gamma(1-s) \Gamma(1-t) \Gamma(1+(s+t))}{\Gamma(1+s) \Gamma(1+t) \Gamma(1-(s+t))} \\
& =-\frac{1}{s t(s+t)} \exp \left[\sum_{k=2}^{\infty} \frac{2 \zeta(2 k+1)}{2 k+1}\left(s^{2 k+1}+t^{2 k+1}-(s+t)^{2 k+1}\right)\right],
\end{aligned}
$$

where the second expression is useful for exhibiting the low-energy expansion. It follows that the first term in this expansion is $P_{8} /(s t u)$, which contains the tree-level supergravity

\footnotetext{
${ }^{12}$ The prefactor $P_{8}$ is the component expression corresponding to the manifestly supersymmetric prefactor $\delta^{16}\left(Q_{4}\right)$ that enters the superamplitude that describes the scattering of any four massless states in the gravity supermultiplet with maximal supersymmetry.
} 
four-particle amplitudes. Once again we will avoid the $t=0$ singularity by subtracting the classical term by defining

$$
\tilde{A}_{c l}(s, t):=A_{c l}(s, t)+\frac{1}{s t(s+t)} .
$$

In this case the amplitude not only has poles on the positive real $s$ axis but also on the positive $u$ (i.e., negative $s$ ) axis and is a special case of the general structure in (2.3). It is easy to see that for $t \leq 0$ this expression has the asymptotic behaviour $\tilde{A}_{c l}(s, t) \underset{s \rightarrow-\infty}{\sim}$ $(-s)^{-2}$ at fixed $t$, which means that the boundary term in (2.9) can be dropped.

We will now express $\tilde{A}_{c l}(s, t)$ as a sum of $s$-channel and $u$-channel poles in the form

$$
\tilde{A}_{c l}(s, t)=\tilde{A}_{c l}^{(s)}(s, t)+\tilde{A}_{c l}^{(u)}(u, t),
$$

making use of the integral representation (which was used in the original paper by Shapiro [28])

$$
\begin{aligned}
\int_{\mathbb{C}} d^{2} w|w|^{-2-2 s}|1-w|^{-2 t} & =-\frac{\pi t}{s(s+t)} \frac{\Gamma(1-s) \Gamma(1-t) \Gamma(1+(s+t))}{\Gamma(1+s) \Gamma(1+t) \Gamma(1-(s+t))} \\
& =\pi t^{2} A_{c l}(s, t) .
\end{aligned}
$$

Dividing the integration domain into the regions (1) $|w| \leq 1$ and (2) $|w| \geq 1$ and using the fact that

$$
\int_{|w| \geq 1} d^{2} w|w|^{-2-2 s}|1-w|^{-2 t}=\int_{|w| \leq 1} d^{2} w|w|^{-2-2 u}|1-w|^{-2 t},
$$

it follows that region (2) is equivalent to region (1) with $s \rightarrow u$.

We now isolate the contributions from the $s$-channel and $u$-channel poles in

$$
\begin{aligned}
\tilde{A}_{c l}^{(s)}(s, t) & :=\frac{1}{\pi t^{2}} \int_{0}^{1} \frac{d r}{r} \int_{0}^{2 \pi} d \theta r^{-2 s}\left(1-r e^{i \theta}\right)^{-t}\left(1-r e^{-i \theta}\right)^{-t}+\frac{1}{t^{2} s} \\
& =\frac{2}{t^{2}} \int_{0}^{1} \frac{d r}{r} \sum_{m=1}^{\infty} r^{-2 s+2 m}\left(\begin{array}{c}
-t \\
m
\end{array}\right)^{2} \\
& =\sum_{m=1}^{\infty} \frac{1}{m-s}\left(\frac{\Gamma(m+t)}{\Gamma(1+t) \Gamma(1+m)}\right)^{2},
\end{aligned}
$$

and

$$
\begin{aligned}
\tilde{A}_{c l}^{(u)}(u, t) & :=\frac{1}{\pi t^{2}} \int_{0}^{1} \frac{d r}{r} \int_{0}^{2 \pi} d \theta r^{2 s+2 t}\left(1-r e^{i \theta}\right)^{-t}\left(1-r e^{-i \theta}\right)^{-t}+\frac{1}{t^{2} u} \\
& =\sum_{m=1}^{\infty} \frac{1}{s+t+m}\left(\frac{\Gamma(m+t)}{\Gamma(1+t) \Gamma(1+m)}\right)^{2},
\end{aligned}
$$

where we have used the fact that

$$
\frac{1}{t s(s+t)}=\frac{1}{t^{2} s}+\frac{1}{t^{2} u} .
$$


Comparing the expressions (4.7) and (4.8) with (3.3), it is apparent that the closed string tree amplitude can be obtained from the open string amplitude by squaring the residue of each pole. This is closely related to the Kawai, Lewellen and Tye (KLT) relation [29] and is the string generalization of the Bern, Carrasco and Johansson (BCJ) double copy [30] relation between gauge theory and gravity.

The positivity conditions on the $s$-channel contribution $\left.\partial_{t}^{q} \tilde{A}_{c l}^{(s)}(s, t)\right|_{t=0}$, follow from the fact that the residues of the massive poles have positive coefficients when expanded in terms of Gegenbauer polynomials. We now need to check the asymptotic forms of $A_{c l}^{(s)}(s, t)$ and $A_{c l}^{(u)}(u, t)$ when $t$ is fixed and $|s| \rightarrow \infty$. We have (setting $r=e^{-y}$ ),

$$
\begin{aligned}
A_{c l}^{(s)}(s, t) & =\frac{1}{\pi t^{2}} \int_{0}^{\infty} d y \int_{0}^{2 \pi} d \theta e^{2 s y}\left(1-e^{-y} e^{i \theta}\right)^{-t}\left(1-e^{-y} e^{-i \theta}\right)^{-t} \\
& =\frac{1}{\pi t^{2}} \int_{0}^{\infty} d y \int_{0}^{2 \pi} d \theta e^{2 s y}\left(1+e^{-2 y}-2 e^{-y} \cos \theta\right)^{-t} \\
& \rightarrow \frac{2^{-t}}{\pi t^{2} s} \int_{0}^{\infty} d \hat{y} \int_{0}^{2 \pi} d \theta e^{-2 \hat{y}}\left((1-\cos \theta)\left(1+\frac{\hat{y}}{s}\right)\right)^{-t} \\
& =\frac{2^{-2 t} \Gamma\left(\frac{1}{2}-t\right)}{\pi^{\frac{1}{2}} s t^{2} \Gamma(1-t)}+\mathcal{O}\left(s^{-2}\right),
\end{aligned}
$$

where we have rescaled $y \rightarrow \hat{y} /(-s)$ to account for the limit $s \rightarrow-\infty$. We have then expanded the factor of $e^{-y}=e^{\hat{y} / s} \sim 1+\hat{y} / s+O\left(s^{-2}\right)$ in the two brackets. We therefore deduce that

$$
\tilde{A}_{c l}^{(s)}(s, t)=A_{c l}^{(s)}(s, t)-\frac{1}{s t^{2}} \underset{s \rightarrow \infty}{\rightarrow} \frac{1}{s t^{2}}\left(\frac{2^{-2 t} \Gamma\left(\frac{1}{2}-t\right)}{\pi^{\frac{1}{2}} \Gamma(1-t)}-1\right)+O\left(s^{-2}\right) .
$$

More generally, the large- $|s|$ expansion of the amplitude has the form $\tilde{A}_{c l}^{(s)}(s, t) \underset{s \rightarrow \infty}{\rightarrow}$ $\sum_{q=0} c_{q} t^{q} s^{-1}$ with $q \geq 0$, where $c_{q}$ is constant. Therefore, the contour integral at $|s| \rightarrow \infty$ in (2.9) vanishes. The $1 / s$ behaviour in (4.10) cancels with the leading term in $A_{c l}^{(u)}(u, t)$ in the complete amplitude. The full amplitude is Regge behaved and behaves as $(-s)^{t-2}$ as $|s| \rightarrow \infty$.

\subsection{The closed-string low-energy expansion coefficients}

In order to discuss the low-energy expansion of the closed-string tree amplitude we first note that the terms of lowest-order in $t$, i.e. the expansion of the $t=0$ amplitude, have the form

$$
\begin{aligned}
\tilde{A}_{c l}(s, 0) & =\tilde{A}_{c l}^{(s)}(s, 0)+\tilde{A}_{c l}^{(u)}(s, 0)=2 \sum_{n=0}^{\infty} \zeta(2 n+3) s^{2 n} \\
& =-\frac{1}{s}(2 \gamma+\psi(1-s)+\psi(1+s)) \\
& =\frac{1}{s^{2}} \sum_{k=1}^{\infty}\left(\frac{1}{k-s}+\frac{1}{k+s}-\frac{2}{k}\right)
\end{aligned}
$$


which is simply a constant plus the sum of $s$-channel and $u$-channel poles. In this case the coefficient of $s^{2 n}$ is $g_{2 n, 0}=2 \zeta(2 n+3)$. The relevant Hankel matrices can be viewed as sub-matrices of the open-string Hankel matrixes, with the even zeta values set to zero and with $\zeta(2 n+1) \rightarrow 2 \zeta(2 n+1)$. This is the result of the single-valued projection [31], which also reflects the KLT relation between open and closed string tree amplitudes (see e.g. [32-34] for recent applications to superstring amplitudes). The positivity of the determinant of the Hankel matrices formed from these coefficients provides no further constraints on products of zeta values beyond those deduced from the open-string case.

\subsection{Low-energy expansion of $\tilde{A}_{c l}^{(s)}(s, t)$}

Unlike the colour-ordered open string amplitudes, the coefficients of low-energy expansion $\tilde{A}_{c l}(s, t)$ are generally not positive definite due to the $u$-channel contribution, Consequently, they do not reside on a moment curve, apart from the $t^{0}$ term discussed earlier. To deal with this issue, we will consider the unitarity constraints on the $s$-channel contribution, $\tilde{A}_{c l}^{(s)}(s, t)$, in (4.7). As we will show below, the coefficients of the low-energy expansion of $\tilde{A}_{c l}^{(s)}(s, t)$ are not only positive, but also satisfy the Hankel matrix constraints, just as in the case of open superstring amplitudes. Interestingly, even though the low-energy expansion of $\tilde{A}_{c l}(s, t)$ only contains powers of single odd zeta values, individually the low-energy coefficients of $\tilde{A}_{c l}^{(s)}(s, t)$ and $\tilde{A}_{c l}^{(u)}(u, t)$ include irreducible MZVs as well as even zeta values. Therefore, the unitary of $\tilde{A}_{c l}^{(s)}(s, t)$ leads to Hankel matrix constraints on irreducible MZVs.

The low energy expansion coefficients. The expansion of $\tilde{A}_{c l}^{(s)}(s, t)$ and $\tilde{A}_{c l}^{(t)}(u, t)$ can be obtained to any given order in the low energy expansion by explicit expansion of (4.7) and (4.8). Motivated by the expressions in [26] we obtain this expansion in the form

$$
\begin{aligned}
\tilde{A}_{c l}^{(s)}(s, t) & =\sum_{n=1}^{\infty} \frac{1}{n-s}\left(\frac{\Gamma(n+t)}{\Gamma(1+t) \Gamma(1+n)}\right)^{2}=\sum_{p=0}^{\infty} s^{p} \sum_{n=1}^{\infty} \frac{1}{n^{p+1}}\left(\frac{\Gamma(n+t)}{\Gamma(1+t) \Gamma(1+n)}\right)^{2} \\
& =\sum_{p=0}^{\infty} s^{p} \sum_{q=0}^{\infty} Z(p+3, q) t^{q}
\end{aligned}
$$

The quantity $Z(p+3, q)$ is defined by the generating function

$$
\sum_{q=0}^{\infty} Z(p+3, q) t^{q}=\sum_{n=1}^{\infty} \frac{1}{n^{p+1}}\left(\frac{\Gamma(n+t)}{\Gamma(1+t) \Gamma(1+n)}\right)^{2}=\sum_{n=1}^{\infty} \frac{1}{n^{p+3}} \prod_{0<m<n}\left(1+\frac{t}{m}\right)^{2}
$$

and can be expressed as a linear sum of elements of a particular class of MZVs of weight $q+r$,

$$
Z(r, q):=\sum_{\substack{q \in\{1,2\}^{j}, j \geq 0 \\ q_{1}+\cdots+q_{j}=q}} 2^{\#\left\{i: q_{i}=1\right\}} \zeta(\mathbf{q}, r)
$$

In this expression the components of the $j$-component vector $\mathbf{q}=\left(q_{1}, q_{2}, \ldots, q_{j}\right)$ are summed over values $q_{i}=1,2$, subject to the condition $\sum_{i=1}^{j} q_{i}=q$. Furthermore, 
$\#\left\{i: q_{i}=1\right\}$ in the coefficient $2^{\#\left\{i: q_{i}=1\right\}}$ denotes the numbers of components with $q_{i}=1$. It follows from (4.13) that the closed-string coefficients are simply given by

$$
g_{p, q}^{c l}=Z(p+3, q) .
$$

Interestingly, as stressed in [26], the quantities $Z(r, q)$ not only arise in the expansion of the tree-level closed-string amplitude, but also in the evaluation of the low-energy expansion of the genus-one four-graviton amplitude [35]. In that context certain multiple sums arise in considering the coefficients in the Laurent polynomial of the $\operatorname{large-\operatorname {Im}} \tau$ expansion of the two-point functions, $D_{\ell}(\tau)$, on a genus-one surface of complex structure $\tau$. These multiple sums have the form

$$
S(m, n) \equiv \sum_{k_{1}, \ldots, k_{m} \neq 0} \frac{\delta\left(\sum_{1 \leq i \leq m} k_{i}\right)}{\left|k_{1} \cdots k_{m}\right|\left(\left|k_{1}\right|+\cdots+\left|k_{m}\right|\right)^{n}} .
$$

In appendix A.3.2 of [35] authored by Don Zagier it was proved that

$$
S(q+2, r-2)=(q+2) ! 2^{2-r} Z(r, q),
$$

with $Z(r, q)$ given by (4.15). The interesting fact that $Z(r, q)$ arises in the low-energy expansion of the genus-one amplitude as well as the expansion of the tree amplitude was emphasised in [26].

The expressions for the coefficients of $t^{0}$ and $t^{1}$ are the following combinations of polynomials in zeta values

$$
\begin{aligned}
& Z(r, 0)=\zeta(r) \\
& Z(r, 1)=2 \zeta(1, r)=r \zeta(r+1)-\sum_{i=1}^{r-2} \zeta(i+1) \zeta(r-i) .
\end{aligned}
$$

We see that the coefficients of the low-energy expansion of $\tilde{A}_{c l}^{(s)}(s, t)$ bear a very close resemblance to the coefficients in the expansion of the open-string amplitude in (3.15). The $q=0$ (i.e. $t^{0}$ ) terms are identical whereas the $q=1$ (i.e. $t^{1}$ ) terms have an additional factor of 2. This fact can be understood from the double copy structure of (4.7) so that the residue of each pole of closed-string amplitude is the square of that of the open string amplitude.

When $q=2$, we have

$$
Z(r, 2)=\zeta(2, r)+4 \zeta(1,1, r),
$$

where $\zeta(1,1, r)$ can always be reduced to zeta values as given in $(3.15)$, and $\zeta(2, r)$ can also be reduced to a polynomial in zeta values when $r<6$. However, when $r=6$ the MZV $\zeta(2,6)$ is irreducible. Indeed, $Z(r, q)$ generally contains irreducible MZVs when $q>1$ and $r+q \geq 8$. The $\tilde{A}_{c l}^{(u)}(u, t)$ part of the complete closed-string amplitude has the same structure as above, but with $s$ replaced by $u=-s-t$. All the even zeta values as well as all the irreducible MZVs cancel in the sum of $\tilde{A}_{c l}^{(s)}(s, t)$ and $\tilde{A}_{c l}^{(u)}(u, t)$ so the full four-particle closed-string tree amplitude can be expressed in terms of odd zeta values only. 
It follows from the earlier discussion that the $n \times n$ Hankel matrix associated with the coefficients of the low-energy expansion of $\tilde{A}_{c l}^{(s)}(s, t)$ has elements given by

$$
H_{n}^{c l(s)}\left[\mathbf{Z}_{\mathbf{q}}\right]_{i j}=Z(i+j+1, q), \quad i, j=1,2, \ldots, n,
$$

An example of a $3 \times 3$ Hankel matrix for any $q$ is given by

$$
H_{3}^{c l(s)}\left[\mathbf{Z}_{\mathbf{q}}\right]=\left(\begin{array}{ccc}
Z(3, q) & Z(4, q) & Z(5, q) \\
Z(4, q) & Z(5, q) & Z(6, q) \\
Z(5, q) & Z(6, q) & Z(7, q)
\end{array}\right)
$$

Much as before, the positivity conditions lead to conditions of the form

$$
\operatorname{det} H_{n}^{c l(s)}\left[\mathbf{Z}_{\mathbf{q}}\right]>0,
$$

as well as a host of such inequalities expressing the positivity of any minor of $H_{n, q}^{c l(s)}[Z]_{i j}$ for all $n \geq 3$. As discussed earlier, when $q \geq 2, Z(i+j+1, q)$ generally contains irreducible MZVs. For example, in the $q=2$ case (4.22) becomes

$$
\begin{aligned}
& H_{3}^{c l(s)}\left[\mathbf{Z}_{\mathbf{2}}\right] \\
& =\left(\begin{array}{ccc}
\frac{5 \zeta(5)}{2}-\zeta(2) \zeta(3) & \frac{53 \zeta(6)}{12}-3 \zeta(3)^{2} & 9 \zeta(7)-3 \zeta(2) \zeta(5)-3 \zeta(3) \zeta(4) \\
\ldots & \ldots & \zeta(2,6)+\frac{61 \zeta(8)}{6}+2 \zeta(2) \zeta(3)^{2}-12 \zeta(3) \zeta(5) \\
\ldots & \ldots & \frac{113 \zeta(9)}{6}-5 \zeta(2) \zeta(7)-5 \zeta(3) \zeta(6)-5 \zeta(4) \zeta(5)+\frac{2 \zeta(3)^{3}}{3}
\end{array}\right)
\end{aligned}
$$

where the ellipsis represent entries that are identified by the fact that the matrix is symmetric. In this case the positivity conditions (4.23) lead to conditions on polynomials that contain the irreducible MZV $\zeta(2,6)$.

\section{Discussion}

As discussed by Arkani-Hamed, Huang and Huang [11-13], general considerations of unitarity and asymptotic behaviour of four-particle scattering amplitudes lead to very interesting geometric constraints on low-energy physics. As a consequence the coefficients in the low-energy expansion must reside inside a cyclic polytope, which is determined by the Gegenbauer polynomials. This leads to a large number of positivity constraints on polynomials of the low energy coefficients that are encoded in the positivity of Hankel matrices and their minors.

In this paper we have explored these positivity constraints on the coefficients of the low-energy expansions of tree-level amplitudes in open and in closed superstring theories. Despite the fact that considerations of the four-particle amplitude with massless external states can only probe a limited amount of information this nevertheless leads to a host of interesting inequalities involving coefficients in the low energy expansion, which are rational polynomials of multiple zeta values. These constraints follow from positivity of the determinants of the Hankel matrix (and any of its minors) formed from these coefficients. The simplest version of these inequalities reproduces the known results in math literature $[23,24]$. Our consideration from unitarity of superstring amplitudes not only provides 
a physical interpretation of the known inequalities among single zeta values, but also leads to a host of new relations among single zeta values as well as more general MZVs. These inequalities are the necessary conditions for superstring amplitudes being unitary, it would be of interest to prove these inequalities by other means.

Another aspect of the positivity properties of the amplitude that was stressed in [11-13] involves reorganising the low-energy expansion so that it takes the form $\sum_{\Delta, q} \tilde{g}_{\Delta, q} s^{\Delta-q} t^{q}$, where $\Delta=p+q$. This leads to positivity conditions on the vector of coefficients, $\mathbf{g}_{\boldsymbol{\Delta}}=$ $\left\{g_{\Delta, 0}, \ldots, g_{\Delta, n}\right\}$ that imply that this vector (for arbitrary $n$ ) must reside inside the cyclic polytope generated by expanding the Gegenbauer polynomials in powers of $t$ [11-13]. It might be interesting to study the implications of these constraints on the MZV coefficients of open and closed string theories.

It is of note that in the case of the closed-string amplitude, the positivity constraints are constraints on rational polynomials of irreducible MZVs. These follow from the introduction of the quantities $Z(r, q)$ (introduced in [26] and defined in (4.15)) that are combinations of MZVs that arise as intermediate coefficients in the low energy expansion of the closed-string four-particle amplitude. Although the irreducible MZVs cancel in the expansion of the full four-particle amplitude, they contribute to the portion of the amplitude that has $s$-channel poles and satisfy the conditions contained in (4.23).

The inequalities satisfied by polynomials in MZVs implied by unitarity of superstring tree amplitudes generalise results in the mathematics literature [23, 24] on determinants of Hankel matrices of single zeta values. The determinants of these $n \times n$ Hankel matrices approach zero very rapidly as a function of $n$. This is easily verified by direct numerical estimation and in the cases with single zeta values the explicit expressions for the asymptotic behaviour are known $[23,24]$ (and are quoted in (3.10) and (3.11)). It would be of interest to determine analogous expressions for the $n$-dependence of the asymptotic behaviour of the determinants of Hankel matrices of MZVs that arise in this paper. It would also be of obvious interest to develop an interpretation of the asymptotic behaviour of such matrices in terms of asymptotic properties of superstring scattering amplitudes.

We know that in order to resolve the full content of the no-ghost theorem [20-22] it is necessary to consider massless $N$-point amplitudes for all values of $N$. This should be possible, given the explicit expressions for such amplitudes in both open and closed superstring theories $[36,37]$. The study of higher-point massless amplitudes or four-point amplitudes with more general massive external states should lead to more general unitarity conditions on the MZVs. Furthermore, the generalisation to amplitudes of higher genus raises interesting new issues relating to the presence of massless threshold singularities that arise in the low energy expansion (such as those discussed in the genus-one case in $[35,38]$ ).

\section{Acknowledgments}

We are grateful to Oliver Schlotterer for useful conversations and to Nima Arkani-Hamed for comments on the draft of this paper. CW would like to thank Yu-tin Huang for collaboration and discussions on related topics. CW is supported by a Royal Society University Research Fellowship No. UF160350. MBG has been partially supported by STFC con- 
solidated grant ST/L000385/1 and by a Leverhulme Emeritus Fellowship. This work was finalised during the workshop "Scattering Amplitudes and the Conformal Bootstrap" at the Aspen Center for Physics, which is supported by National Science Foundation grant PHY-1607611, and was partially supported by a grant from the Simons Foundation.

\section{A Four-particle superstring tree amplitudes at higher orders in $t$}

For completeness, we here present the expansion of the tree-level four-point functions at the first few orders in $s$ and $t$. In the case of the open string expression (3.3) this expansion has the form (up to $O\left(t^{3}\right)$ and $O\left(s^{5}\right)$ )

$$
\begin{aligned}
& \tilde{A}_{o p}(s, t)=\zeta(2)+s \zeta(3)+s^{2} \zeta(4)+s^{3} \zeta(5)+s^{4} \zeta(6)+s^{5} \zeta(7)+O\left(s^{6}\right) \\
& +t\left(\zeta(3)+\frac{\zeta(4)}{4} s+(2 \zeta(5)-\zeta(2) \zeta(3)) s^{2}+\left(\frac{3 \zeta(6)}{4}-\frac{\zeta(3)^{2}}{2}\right) s^{3}\right. \\
& \left.\quad+(3 \zeta(7)-\zeta(4) \zeta(3)-\zeta(2) \zeta(5)) s^{4}+\left(\frac{5 \zeta(8)}{4}-\zeta(3) \zeta(5)\right) s^{5}+O\left(s^{6}\right)\right) \\
& +t^{2}\left(\zeta(4)+(2 \zeta(5)-\zeta(2) \zeta(3)) s+\left(\frac{23 \zeta(6)}{16}-\zeta(3)^{2}\right) s^{2}\right. \\
& \quad+\left(5 \zeta(7)-\frac{5 \zeta(4) \zeta(3)}{4}-2 \zeta(2) \zeta(5)\right) s^{3}+\left(\frac{61 \zeta(8)}{24}+\frac{\zeta(2) \zeta(3)^{2}}{2}-3 \zeta(5) \zeta(3)\right) s^{4} \\
& \left.\quad+\left(\frac{\zeta(3)^{3}}{6}-\frac{7}{4} \zeta(6) \zeta(3)-\frac{9}{4} \zeta(4) \zeta(5)-3 \zeta(2) \zeta(7)+\frac{28 \zeta(9)}{3}\right) s^{5}+O\left(s^{6}\right)\right) \\
& +t^{3}\left(\zeta(5)+\left(\frac{3 \zeta(6)}{4}-\frac{\zeta(3)^{2}}{2}\right) s+\left(5 \zeta(7)-\frac{5}{4} \zeta(4) \zeta(3)-2 \zeta(2) \zeta(5)\right) s^{2}\right. \\
& +\left(\zeta(2) \zeta(3)^{2}-4 \zeta(5) \zeta(3)+\frac{499 \zeta(8)}{192}\right) s^{3}+\left(\frac{\zeta(3)^{3}}{2}-\frac{35}{16} \zeta(6) \zeta(3)-\frac{7}{2} \zeta(4) \zeta(5)-5 \zeta(2) \zeta(7)\right. \\
& \left.+14 \zeta(9)) s^{4}+\left(\frac{9}{8} \zeta(4) \zeta(3)^{2}+3 \zeta(2) \zeta(5) \zeta(3)-8 \zeta(7) \zeta(3)-4 \zeta(5)^{2}+\frac{973 \zeta(10)}{160}\right) s^{5}+O\left(s^{6}\right)\right) .
\end{aligned}
$$

The expansion of the closed-string expression $\tilde{A}_{c l}^{(s)}(s, t)$ up to $O\left(t^{2}\right)$ and $O\left(s^{4}\right)$ has the form

$$
\begin{aligned}
& \tilde{A}_{c l}^{(s)}(s, t)=\zeta(3)+s \zeta(4)+s^{2} \zeta(5)+s^{3} \zeta(6)+s^{4} \zeta(7)+O\left(s^{5}\right) \\
& +2 t\left(\frac{\zeta(4)}{4}+(2 \zeta(5)-\zeta(2) \zeta(3)) s+\left(\frac{3 \zeta(6)}{4}-\frac{\zeta(3)^{2}}{2}\right) s^{2}\right. \\
& \left.+(3 \zeta(7)-\zeta(4) \zeta(3)-\zeta(2) \zeta(5)) s^{3}+\left(\frac{5 \zeta(8)}{4}-\zeta(3) \zeta(5)\right) s^{4}+O\left(s^{5}\right)\right) \\
& +t^{2}\left(\frac{5 \zeta(5)}{2}-\zeta(2) \zeta(3)+\left(\frac{53 \zeta(6)}{12}-3 \zeta(3)^{2}\right) s+(9 \zeta(7)-3 \zeta(2) \zeta(5)-3 \zeta(3) \zeta(4)) s^{2}\right. \\
& +\left(\zeta(2,6)+\frac{61 \zeta(8)}{6}+2 \zeta(2) \zeta(3)^{2}-12 \zeta(3) \zeta(5)\right) s^{3} \\
& \left.+\left(\frac{113 \zeta(9)}{6}-5 \zeta(2) \zeta(7)-5 \zeta(3) \zeta(6)-5 \zeta(4) \zeta(5)+\frac{2 \zeta(3)^{3}}{3}\right) s^{4}+O\left(s^{5}\right)\right) .
\end{aligned}
$$


The low-energy expansion of $\tilde{A}_{c l}^{(u)}(u, t)$ is the same with $s$ and $u=-t-s$ interchanged. Each of these expressions contains even zeta values and irreducible MZVs - for example, the coefficient of $t^{2} s^{3}$ in (A.2) contains the weight-8 irreducible MZV $\zeta(2,6)$. These cancel out in the low-energy expansion of the total closed-string tree amplitude, $\tilde{A}_{c l}(s, t)=\tilde{A}_{c l}^{(s)}(s, t)+$ $\tilde{A}_{c l}^{(u)}(u, t)$, which has coefficients that are rational polynomials of odd zeta values.

Open Access. This article is distributed under the terms of the Creative Commons Attribution License (CC-BY 4.0), which permits any use, distribution and reproduction in any medium, provided the original author(s) and source are credited.

\section{References}

[1] G. Veneziano, Construction of a crossing-symmetric, Regge behaved amplitude for linearly rising trajectories, Nuovo Cim. A 57 (1968) 190 [InSPIRE].

[2] M.A. Virasoro, Alternative constructions of crossing-symmetric amplitudes with Regge behavior, Phys. Rev. 177 (1969) 2309 [InSPIRE].

[3] G. 't Hooft, A planar diagram theory for strong interactions, Nucl. Phys. B 72 (1974) 461 [INSPIRE].

[4] A. Adams, N. Arkani-Hamed, S. Dubovsky, A. Nicolis and R. Rattazzi, Causality, analyticity and an IR obstruction to UV completion, JHEP 10 (2006) 014 [hep-th/0602178] [INSPIRE].

[5] Z. Komargodski and A. Schwimmer, On renormalization group flows in four dimensions, JHEP 12 (2011) 099 [arXiv:1107.3987] [INSPIRE].

[6] N. Arkani-Hamed, L. Motl, A. Nicolis and C. Vafa, The string landscape, black holes and gravity as the weakest force, JHEP 06 (2007) 060 [hep-th/0601001] [INSPIRE].

[7] C. Cheung and G.N. Remmen, Infrared consistency and the weak gravity conjecture, JHEP 12 (2014) 087 [arXiv: 1407.7865] [INSPIRE].

[8] S. Andriolo, D. Junghans, T. Noumi and G. Shiu, A tower weak gravity conjecture from infrared consistency, Fortsch. Phys. 66 (2018) 1800020 [arXiv: 1802.04287] [INSPIRE].

[9] Y. Hamada, T. Noumi and G. Shiu, Weak gravity conjecture from unitarity and causality, Phys. Rev. Lett. 123 (2019) 051601 [arXiv:1810.03637] [INSPIRE].

[10] B. Bellazzini, M. Lewandowski and J. Serra, Amplitudes' positivity, weak gravity conjecture and modified gravity, arXiv:1902.03250 [INSPIRE].

[11] N. Arkani-Hamed, T.-Z. Huang and Y.-T. Huang, in preparation.

[12] N. Arkani-Hamed, Positive geometry of effective field theory, lectures at CERN Winter School on Supergravity, Strings and Gauge Theory, CERN, Geneva, Switzerland, 4-8 February 2019.

[13] Y.-T. Huang, The space of EFT and CFT: life behind the facets of cyclic polytopes, in Amplitudes 2018, SLAC, U.S.A., 19 June 2018.

[14] C. de Rham, S. Melville, A.J. Tolley and S.-Y. Zhou, Positivity bounds for scalar field theories, Phys. Rev. D 96 (2017) 081702 [arXiv: 1702.06134] [INSPIRE].

[15] C. de Rham, S. Melville and A.J. Tolley, Improved positivity bounds and massive gravity, JHEP 04 (2018) 083 [arXiv:1710.09611] [INSPIRE].

[16] W.-M. Chen, Y.-T. Huang, T. Noumi and C. Wen, Unitarity bounds on charged/neutral state mass ratios, Phys. Rev. D 100 (2019) 025016 [arXiv:1901.11480] [INSPIRE]. 
[17] N. Arkani-Hamed, Y.-T. Huang and S.-H. Shao, On the positive geometry of conformal field theory, JHEP 06 (2019) 124 [arXiv: 1812.07739] [INSPIRE].

[18] K. Sen, A. Sinha and A. Zahed, Positive geometry in the diagonal limit of the conformal bootstrap, arXiv: 1906.07202 [INSPIRE].

[19] S. Fallat, C.R. Johnson and A.D. Sokal, Total positivity of sums, Hadamard products and Hadamard powers: results and counterexamples, Linear Alg. Appl. 520 (2017) 242 [arXiv: 1612.02210].

[20] R.C. Brower, Spectrum generating algebra and no ghost theorem for the dual model, Phys. Rev. D 6 (1972) 1655 [INSPIRE].

[21] P. Goddard and C.B. Thorn, Compatibility of the dual Pomeron with unitarity and the absence of ghosts in the dual resonance model, Phys. Lett. B 40 (1972) 235 [INSPIRE].

[22] C.B. Thorn, A proof of the no-ghost theorem using the Kac determinant, MSRI Publ. 3 (1985) 411 [INSPIRE].

[23] H. Monien, Hankel determinants of Dirichlet series, arXiv:0901.1883.

[24] A. Haynes and W. Zudilin, Hankel determinants of zeta values, SIGMA 11 (2015) 101 [arXiv: 1510.01901].

[25] R.J. Eden, P.V. Landshoff, D.I. Olive and J.C. Polkinghorne, The analytic S-matrix, Cambridge University Press, Cambridge, U.K. 1966.

[26] D. Zagier and F. Zerbini, Genus-zero and genus-one string amplitudes and special multiple zeta values, arXiv:1906.12339 [INSPIRE].

[27] M.E. Hoffman, The algebra of multiple harmonic series, J. Alg. 194 (1997) 477.

[28] J.A. Shapiro, Electrostatic analog for the Virasoro model, Phys. Lett. B 33 (1970) 361 [INSPIRE].

[29] H. Kawai, D.C. Lewellen and S.-H. Henry Tye, A relation between tree amplitudes of closed and open strings, Nucl. Phys. B 269 (1986) 1 [INSPIRE].

[30] Z. Bern, J.J.M. Carrasco and H. Johansson, New relations for gauge-theory amplitudes, Phys. Rev. D 78 (2008) 085011 [arXiv: 0805.3993] [INSPIRE].

[31] F. BROWN, Single-valued motivic periods and multiple zeta values, Forum Math. Sigma 2 (2014) e25.

[32] F. Brown and C. Dupont, Single-valued integration and double copy, arXiv:1810.07682 [INSPIRE].

[33] O. Schlotterer and O. Schnetz, Closed strings as single-valued open strings: a genus-zero derivation, J. Phys. A 52 (2019) 045401 [arXiv:1808.00713] [INSPIRE].

[34] P. Vanhove and F. Zerbini, Closed string amplitudes from single-valued correlation functions, arXiv: 1812.03018 [INSPIRE].

[35] M.B. Green, J.G. Russo and P. Vanhove, Low energy expansion of the four-particle genus-one amplitude in type-II superstring theory, JHEP 02 (2008) 020 [arXiv:0801.0322] [INSPIRE].

[36] O. Schlotterer and S. Stieberger, Motivic multiple zeta values and superstring amplitudes, J. Phys. A 46 (2013) 475401 [arXiv:1205.1516] [InSPIRE].

[37] C.R. Mafra, O. Schlotterer and S. Stieberger, Complete N-point superstring disk amplitude I. Pure spinor computation, Nucl. Phys. B 873 (2013) 419 [arXiv:1106.2645] [INSPIRE].

[38] E. D'Hoker and M.B. Green, Exploring transcendentality in superstring amplitudes, JHEP 07 (2019) 149 [arXiv:1906.01652] [INSPIRE]. 